\title{
Preparation and characterization of a Polyacrylonitrile based gel polymer electrolyte for redox capacitors
}

\author{
C.M. Bandaranayake, W.A.D.S.S. Weerasinghe, K.P. Vidanapathirana and \\ K.S. Perera ${ }^{1}$ \\ Department of Electronics, Wayamba University of Sri Lanka, Kuliyapitiya, Sri Lanka \\ Correspondence: ${ }^{1}$ kumudu31966@gmail.com
}

Received: $17^{\text {th }}$ February 2016, Revised: $23^{\text {rd }}$ June 2016, Accepted: $24^{\text {th }}$ June 2016

\begin{abstract}
In this study, a gel polymer electrolyte (GPE) consisting with polyacrylonitrile (PAN), ethylene carbonate (EC), propylene carbonate (PC) and magnesium trifluromethane sulfonate $\left(\mathrm{Mg}\left(\mathrm{CF}_{3} \mathrm{SO}_{3}\right)_{2}\right)$ was prepared using the hot pressed method. The starting materials were heated at $130{ }^{\circ} \mathrm{C}$ for 2 hours and the resulting hot viscous mixture was pressed in between two well cleaned glass plates. The composition was fine-tuned by varying the salt and the polymer concentration in order to obtain a mechanically stable, thin and flexible film with a high ionic conductivity. It was found that the composition, $105 \mathrm{PAN}: 150 \mathrm{MgTF}$ : $400 \mathrm{EC}: 400 \mathrm{PC}$ gives the maximum conductivity of $1.06 \times 10^{-2} \mathrm{Scm}^{-1}$. DC polarization test done with blocking electrodes confirmed the ionic nature of the sample while the results obtained with non-blocking electrodes proved that the anionic contribution for the conductivity is dominant. The sample was used in redox capacitors having two identical polypyrrole electrodes doped with dodecylbenzesulfonate. Cyclic Voltammetry, Galvanostatic Charge Discharge and Electrochemical Impedance Spectroscopy techniques were used to evaluate the performance of the redox capacitors. The specific capacitance was high at low scan rates. The electrolyte was quite stable when use in the redox capacitors. Further, redox capacitor was having a good cycleability which is one of the important key issues to be considered for practical applications.
\end{abstract}

Keywords. Gel polymer electrolyte, Redox capacitor, Polyacrylonitrile, Polypyrrole, EIS.

\section{Introduction}

Basically, a gel polymer electrolyte (GPE) consists of a polymer network, a salt and solvent/s. So, they exhibit higher ambient temperature ionic conductivities comparable to liquid electrolytes and good mechanical 
properties similar to solid electrolytes. In addition, they possess some several unique properties like ease of preparation, wide range of composition with control properties, good adhesive properties and satisfactory thermal and electrical stability. The role of the polymer network is very important as it envelopes the liquid part containing the salt and the solvent/s and thereby prevents their escape. Due to that, GPEs do not undergo leakage problems suffer by liquid electrolytes. Hence, GPEs have been recognized as better alternatives for liquid electrolytes in a wide range of applications such as batteries, electrochromic devices, dye sensitized solar cells, artificial muscles and super capacitors (Liu et al. 2014; Perera et al. 2007; Wu et al. 2013).

Today, energy storage devices have received a global attention due to predicted power crisis that may come up in the next few decades. Super capacitors are thought as one class of potential energy storage devices to be employed in hybrid automobiles, digital telecommunication etc. There are two types of super capacitors namely electrochemical double layer capacitors (EDLCs) and redox capacitors (Wang et al. 2015). EDLCs use carbon based materials as electrodes. In redox capacitors, electro active materials such as conducting polymers and metal oxides are used as electrodes and they exhibit capacitive behaviour due to redox (faradaic) reactions when assembled with a suitable electrolyte. At the moment, many of the reports about redox capacitors are based on liquid electrolytes (Ingram et al. 2004; Hashmi 2004). But, they have undergone different disadvantages such as leakage, corrosion and bulky design. Hence, use of gel polymer electrolytes in place of liquid electrolytes has emerged as a novel concept in the field of energy storage devices.

In this study, a GPE consisted with polyacrylonitrile (PAN), ethylene carbonate (EC), propylene carbonate (PC) and magnesium trifluromethane sulfonate $\left(\mathrm{Mg}\left(\mathrm{CF}_{3} \mathrm{SO}_{3}\right)_{2}\right)$ was prepared by fine tuning the composition to optimize the ionic conductivity. The properties of the sample having the highest room temperature conductivity and good mechanical stability were investigated and then, its potential candidacy in conducting polymer based redox capacitors was evaluated.

\section{Materials and Methods}

\subsection{Preparation of gel polymer electrolytes}

Starting materials polyacrylonitrile (PAN) (ALDRICH,MW 150000), ethylene carbonate (EC)(ALDRICH, 98\%), propylene carbonate (PC)(ALDRICH, 99\%) and magnesium trifluromethane sulfonate $\left(\mathrm{Mg}\left(\mathrm{CF}_{3} \mathrm{SO}_{3}\right)_{2}, \mathrm{MgTF}\right)(\mathrm{ALDRICH}, 99 \%)$ were used as received. To prepare 
the samples, hot-pressed method was used. First, the required amount of PAN was dissolved in a mixture of EC/PC. The weight ratio of EC and PC was kept at 1:1. After performing magnetic stirring for about 2 hours, appropriate amount of MgTF was mixed in. Heating was done at $130{ }^{\circ} \mathrm{C}$ for about 2 hours. The resulting hot viscous mixture was pressed in between two well cleaned glass plates and vacuum dried at room temperature overnight. This procedure was repeated by varying salt concentration as well as polymer concentration. Preparation steps were carried out in side an Argon filled glove box.

\subsection{AC conductivity measurements}

Electrochemical Impedance Spectroscopy (EIS) is a powerful analytical technique to gather information about the resistive and capacitive properties of materials. In this method, properties of materials are analysed using the impedance data gathered by varying the frequency.

A circular shape electrolyte sample having a diameter of $14 \mathrm{~mm}$ was sandwiched in between two stainless steel (SS) electrodes inside a spring loaded brass sample holder which is sealed by means of an $\mathrm{O}$ ring. AC impedance data collection was accomplished in the frequency range $0.01 \mathrm{~Hz}$ to $0.4 \mathrm{MHz}$ at room temperature using Metrohm M101 impedance analyser. This was repeated for each GPE sample having different salt and polymer concentrations. The room temperature conductivity, $\sigma$ was calculated using the equation, $\sigma=\left(1 / \mathrm{R}_{\mathrm{b}}\right)(\mathrm{t} / \mathrm{A})$ where $\mathrm{R}_{\mathrm{b}}$ is the bulk resistance, $\mathrm{t}$ is the thickness and $A$ is the area of cross section of the GPE. $R_{b}$ was determined by analysing the impedance data. $t$ and diameter of the sample were measured using a micrometer screw gauge. For the composition having the maximum conductivity at room temperature, impedance measurements were taken from room temperature up to $55{ }^{\circ} \mathrm{C}$ in the frequency range $0.01 \mathrm{~Hz}$ to $0.4 \mathrm{MHz}$. Conductivity at each temperature was calculated using the above equation The same composition was used for further investigations.

\subsection{Transference number measurements}

First, a circular shape electrolyte sample was loaded inside a brass sample holder in between two SS electrodes. A DC bias voltage of $1 \mathrm{~V}$ was applied and the current variation with time was monitored. Ionic transference number, $t_{i}$ was calculated using the equation, $t_{i}=\left(I_{i}-I_{S}\right) / I_{i}$ where $I_{i}$ is the initial current and $I_{s}$ is the steady state current. Same procedure was repeated using two magnesium electrodes. The cationic transference number was evaluated using the equation, $t_{+}=I_{s} / I_{i}$. Here, $I_{i}$ is the initial current and $I_{s}$ is the steady state current. 


\subsection{Preparation of conducting polymer electrodes}

Pyrrole (ALDRICH) which has been stored in the dark in a refrigerator was electrochemically polymerized in the presence of sodium dodecylbenzenesulfonate (NaDBS)( ALDRICH, 98\%) on fluorine tin oxide (FTO) glass plates by electrochemical polymerization using a computer controlled potentiostat (Metrohm Autolab 101). The concentration of the monomer, NaDBS was $0.1 \mathrm{M}$. The three electrode electrochemical cell used for electrochemical polymerization was consisted of a working electrode (FTO), a Ag/Agcl reference electrode and a Pt counter electrode. Polypyrrole (PPy) films of the thickness of $1 \times 10^{-6} \mathrm{~m}$ were prepared by applying a current density of $1 \mathrm{~mA} \mathrm{~cm}^{-2}$.

\subsection{Fabrication and characterization of redox capacitors}

Symmetrical redox capacitors of the configuration, PPy:DBS / GPE / PPy:DBS was assembled inside a glove box purged with Ar. Cross sectional area of the assembly was $1 \mathrm{~cm}^{2}$. Cyclic Voltammetry technique was used to study the withstanding of the redox capacitor for continuous cycling at a constant scan rate of $5 \mathrm{mVs}^{-1}$. In the three electrode setup, one PPy : DBS electrode was taken as working electrode whilst the other electrode was the (reference + counter) electrodes. The redox capacitor was cycled in the potential window $-2.4 \mathrm{~V}$ to $2.4 \mathrm{~V}$. The specific capacitance was calculated using the equation, $\mathrm{C}_{\mathrm{s}}=2(\mathrm{JIVV}) / \mathrm{m}(\Delta \mathrm{V}) \mathrm{S}$ (Bandaranayake et al. 2015). Here $\int \mathrm{IdV}$ is the area of integral under the cyclic volatmmogram, $\mathrm{m}$ is the single electrode mass, $\Delta \mathrm{V}$ is the potential window and $\mathrm{S}$ is the scan rate. Redox capacitor was subjected to galvanostatic charging and discharging in the potential window $0.0 \mathrm{~V}-1.5 \mathrm{~V}$. The constant current was set to $2 \times 10^{-4} \mathrm{~A}$. The discharge capacitance was calculated using the equation, $\mathrm{C}_{\mathrm{d}}=(2 \mathrm{I}) /$ $\mathrm{m}(\mathrm{dV} / \mathrm{dt})$ [Wang et al. 2013]. Here, I is the constant current, $\mathrm{m}$ is the single electrode mass, $\mathrm{dV}$ is the discharge potential and $\mathrm{dt}$ is the discharge time. Impedance data were collected in the frequency range from $0.01 \mathrm{~Hz}$ to 0.4 $\mathrm{MHz}$ at the room temperature for the redox capacitor to investigate the resistive and capacitive properties as well as the specific capacitance. For the low frequency region, there exists a relationship between specific capacitance, $\mathrm{C}_{\mathrm{s}}, \mathrm{Z}$ " at low frequency region and $\mathrm{f}$ as $\mathrm{Z} "=1 /\left(2 \pi \mathrm{fC}_{\mathrm{s}}\right)$ [Pandey et al. 2013] which was used to calculate $\mathrm{C}_{\mathrm{s}}$.

\section{Results and Discussion}

Figure 1 shows the variation of room temperature conductivity with the salt concentration for the GPE. The conductivity first increases until it reaches the 
maximum when the concentration is $150 \mathrm{mg}$ and then decreases with the increase of salt concentration further.

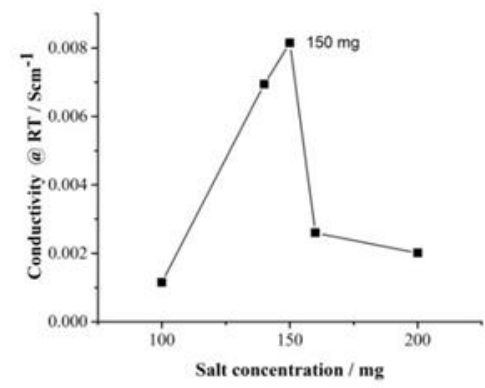

Fig. 1. Variation of room temperature conductivity with salt concentration

The initial increment can be attributed essentially to an increase of free ions which are contributed for conductivity (Jayathilake et al. 2015). When the salt concentration increases further (after the maximum), there is a tendency towards formation of ion aggregates. This reduces the availability of free mobile ions which subsequently reduces conductivity (Ravindran et al. 2012).

Room temperature conductivity was measured by varying the polymer concentration and it is given in Fig.2

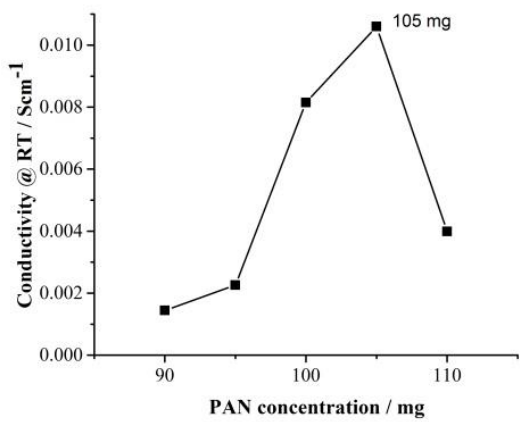

Fig. 2. Variation of room temperature conductivity with polymer concentration.

In Figure 2, it is seen an increment of conductivity initially with increasing PAN concentration followed by a decrement of conductivity with further increment of PAN concentration. This is an indication of a simultaneous occurrence of two competing processes. They are free ion concentration at low PAN concentration and low mobility of ions at high PAN concentration (Sharma et al. 2006; Perera et al. 2011). It was found out that the composition that exhibits the maximum conductivity at room temperature was 105 PAN : $150 \mathrm{MgTF}$ : $400 \mathrm{EC}$ : 400 PC (by weight basis). The value of the conductivity at the maximum is $1.06 \times 10^{-2} \mathrm{Scm}^{-1}$. 
Fig. 3 shows one of the resulting Nyquist plots of the optimized composition at the room temperature. For a cell assembly with the configuration, SS / GPE / SS, the Nyquist plot should contain two semicircles at high and intermediate frequencies while a spike appears at low frequency region. The semicircle at high frequency region corresponds to the bulk electrolyte and the other semicircle represents the charge transfer process at the electrode/electrolyte interface (ayathilake et al. 2014). The spike appears due to the capacitive behaviour of electrodes. In the resulting plot (Fig.3), the high frequency semicircle is absent. This may be due to the unavailability of the required high frequency range in the study $(>0.4 \mathrm{MHz}$ ) (Ramesh et al. 2012). The first intercept on the real axis was used to calculate the conductivity, $\sigma$.

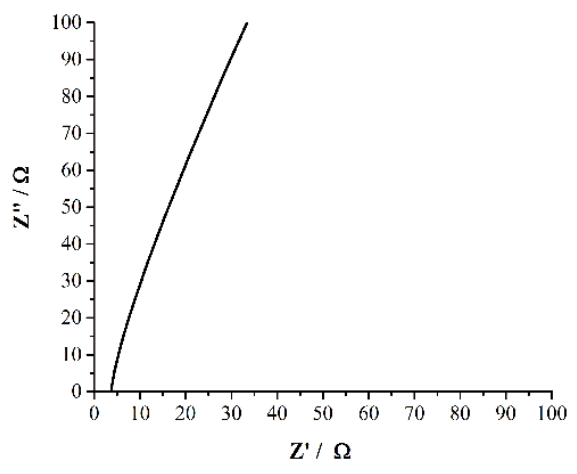

Fig. 3. The resulting Nyquist plot obtained at the room temperature for the GPE of the composition. $105 \mathrm{PAN}$ : $150 \mathrm{MgTF}$ : $400 \mathrm{EC}$ : $400 \mathrm{PC}$

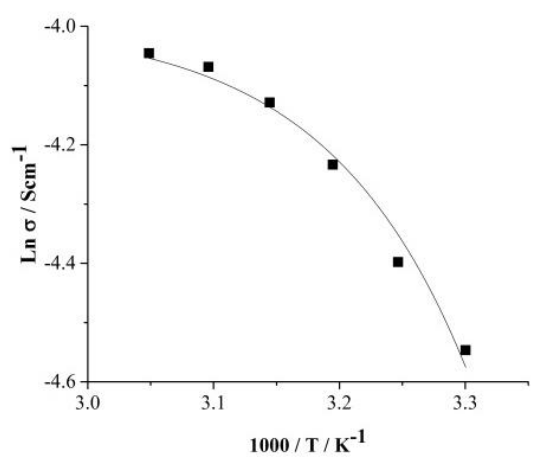

Fig. 4. Variation of conductivity with reciprocal temperature for the GPE of the composition, $105 \mathrm{PAN}: 150 \mathrm{MgTF}$ : $400 \mathrm{EC}: 400 \mathrm{PC}$

Figure 4 shows the $\ln \sigma$ variation with reciprocal temperature. The data could be fitted with Vogel-Tamman-Fulcher (VTF) equation, $\sigma=\mathrm{AT}^{-1 / 2} \exp \left(-\mathrm{E}_{\mathrm{a}} /\right.$ $\left(\mathrm{T}-\mathrm{T}_{0}\right)$ where $\mathrm{A}$ is a pre-exponential factor, $\mathrm{T}$ is the absolute temperature and To is related to the glass transition temperature. This clues that conductivity 
behaviour of the system with the temperature takes places via the free volume formed inside the polymer network (Jayathilake et al. 2014).

Figure 5 exhibits the current variation with time recorded with SS electrodes that are acting as blocking electrodes for mobile ions.

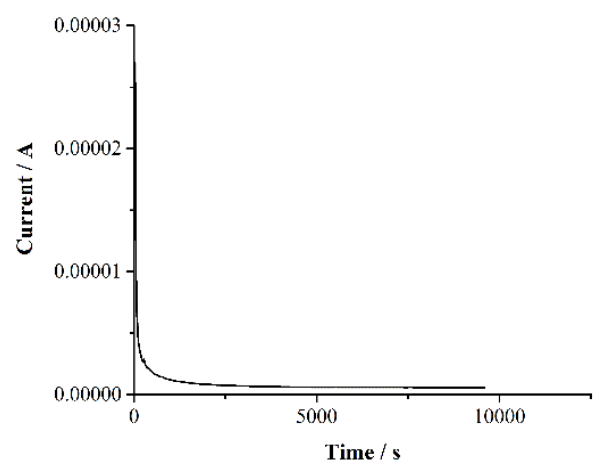

Fig. 5. Variation of current with time for the configuration SS / 105 PAN : 150 MgTF : $400 \mathrm{EC}: 400 \mathrm{PC} / \mathrm{SS}$ at room temperature

An abrupt current decrement could be observed initially followed by a steady state current (Fig. 5). That initial sudden drop is due to polarization of ionic species at the electrolyte/electrode interface. The steady state current arises due to electrons. The calculated value of $\mathrm{t} i=0.96$ ascertains the dominance of ionic charge transport over electron transport in the electrolyte (Kumar et al. 2010). This is highly essential to use the GPE as an electrolyte.

The results of the DC polarization test done with two $\mathrm{Mg}$ electrodes are shown in Fig. 6.

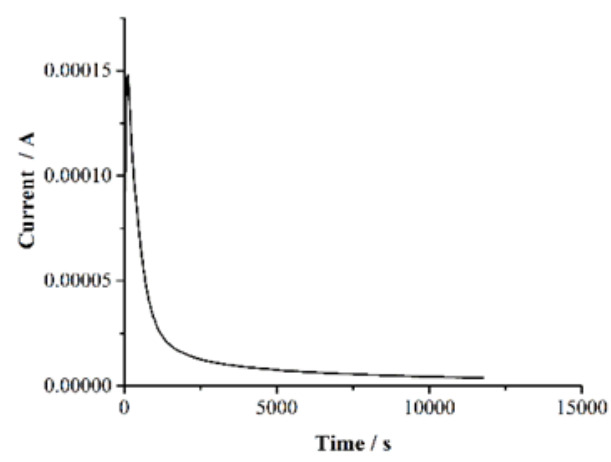

Fig. 6. Variation of current with time for the configuration Mg / 105 PAN : $150 \mathrm{MgTF}$ : $400 \mathrm{EC}: 400 \mathrm{PC} / \mathrm{Mg}$ at room temperature

The cationic and anionic contribution for the conductivity was evaluated using the resultant polarization graph shown in Fig.6. The initial current drop is not 
very steep. Due to $\mathrm{Mg}$ ions for which the electrodes are non-blocking, current has reached the steady state soon. The value of $t_{+}$, which is 0.30 , implies the fact that anion contribution is dominant than cation contribution for the conductivity.

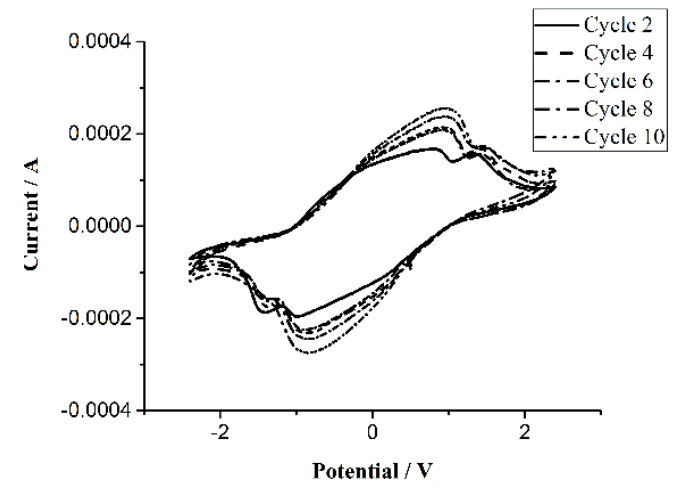

Fig. 7. Cyclic Voltammogram of the redox capacitor in the configuration, PPy : DBS / 105 PAN : $150 \mathrm{MgTF}$ : $400 \mathrm{EC}$ : $400 \mathrm{PC}$ / PPy : DBS at room temperature

In Fig.7, the resulting peaks are corresponding for reduction/oxidation redox reactions. The cyclic volatmmogram with such peaks also reflects the potential dependence of capacitance (Hashmi et al. 2005). The mirror image around $0 \mathrm{~V}$ potential is a characteristic of better reversibility. The average specific capacitance calculated from the CVs is $151.67 \mathrm{Fg}^{-1}$. CVs obtained for each cycle shows similar shape as well as equal area. This is an indication for the presence of a stable specific capacitance over continuous cycling. In other words, results prove the distinctive cycleability of the redox capacitor. Current values in a wide potential window demonstrate the behaviour of the GPE/PPy as charge-storing electrodes in the GPE medium for capacitor application.

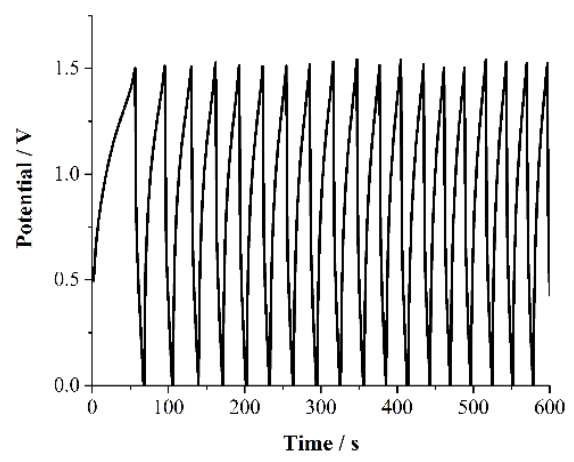

Fig. 8. Galvanostatic charge-discharge curves of a redox capacitor, PPy : DBS / 105 PAN : $150 \mathrm{MgTF}$ : $400 \mathrm{EC}$ : 400 PC / PPy : DBS at room temperature 
The redox capacitors were subjected to charge and discharge under a constant current of $2 \times 10^{-4} \mathrm{~A}$. This value was extracted from the maximum value of current in the resulting CV. Figure 8 shows the continuous charge discharge behaviour for several cycles.

Though the investigation was carried out for 1000 cycles, only few cycles are shown in the figure. The charge-discharge curves are nearly linear. The average specific capacitance was $8 \mathrm{Fg}^{-1}$ which is lower than the value obtained from the cyclic voltammetry technique. This deviation may be due to the difference in scan rates used in the two techniques. The cyclic voltammetry technique was carried out at a scan rate of $5 \mathrm{mVs}^{-1}$. But, for charge discharge test, the calculated value of the scan rate is about $100 \mathrm{mVs}^{-1}$. When the scan rate increases, the specific capacitance reduces as the ions can only partially penetrate into the inner surface of electrodes (Sun et al. 2012 ; Uppugalla et al. 2014). With that explanation, the value obtained at $5 \mathrm{mVs}^{-1}$ should be nearly 20 times higher than the value obtained at $100 \mathrm{mVs}^{-1}$. Accordingly, the two capacitance values $\left(151.67 \mathrm{Fg}^{-1}\right.$ and $\left.8 \mathrm{Fg}^{-1}\right)$ are agreed subject to the difference of scan rate.

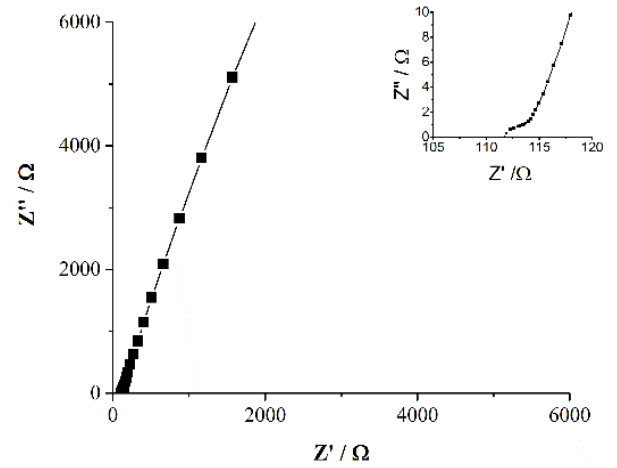

Fig. 9. A resulting Nyqusit plot of a redox capacitor

EIS is a good technique to learn about the characteristic frequency responses of redox capacitors. Figure 9 shows the Nyquist plot obtained for the redox capacitor investigated in the present study. In general, a Nyquist plot of a conventional capacitor should consist of a vertical line parallel to the imaginary impedance axis. But, this is not so under normal situations. Hence, it consists with numerous features at different frequency regions. At high frequency region, a semi-circle appears representing the bulk electrolyte. Another semicircle appears at intermediate frequency region due to the charge transfer resistance and associating double layer capacitance. At lower frequencies the capacitive behaviours become dominant. A tilted line can be seen due to the capacitance related to Warburg diffusion and it will be followed by another tilted line having a steep rise representing the capacitive behaviour of the electrodes. The line having the steep rising behaviour is not perfectly parallel to imaginary axis of impedance as for a typical capacitive 
behaviour. It may be due to the surface roughness as well as non-uniform active layer thickness (Prabhaharan et al. 2006). In the resulting Nyquist plot, the semi-circle corresponding to the bulk electrolyte is absent. It may be due to the unavailability of required high frequency values. The observed bulk electrolyte resistance from the Nyquist plot was used to calculate the bulk electrolyte conductivity and it was in the order of $10^{-2} \mathrm{Scm}^{-1}$ which is comparable with the value obtained with SS electrodes. This is an indication for the stable nature of GPE in redox capacitors. The depressed semicircle in the intermediate frequency range can be attributed for irregularities of electrode surfaces. The two tilted lines with different slopes are present and the calculated specific capacity value was about $2 \mathrm{Fg}^{-1}$ which is quite closer to the value obtained with continuous charge discharge test.

\section{Conclusions}

Polymer and salt concentration affects the conductivity and the mechanical properties of the GPE. The highest conductivity at room temperature, $1.06 \mathrm{x}$ $10^{-2} \mathrm{Scm}^{-1}$, could be obtained with the composition, 105 PAN : $150 \mathrm{MgTF}$ : 400 EC : 400 PC. Furthermore, it was possible to obtain a bubble free, thin and flexible film from that composition. The GPE is predominantly an anionic conductor. The results obtained for redox capacitors confirm the followings.

i. Conducting properties of the GPE remained unchanged when employed in redox capacitors

ii. GPE can withstand for continuous cycling maintaining a constant specific capacitance

iii. Specific capacity varies proportionally with the scan rate

iv. GPE considered in the present study is a suitable candidate to be used in redox capacitors.

Further studies are being carried out to improve the performance.

Acknowledgments. Authors wish to acknowledge National Research Council, Sri Lanka (NRC 12-109), University Grants Commission, Sri Lanka (UGC/VC/DRIC/IRG-2014/WUSL) and National Science Foundation, Sri Lanka (RG/2014/BS/01) for their financial assistance.

\section{References}

Bandaranayake CM, Weerasinghe WADSS, Vidanapathirana KP, Perera KS 2015. A cyclic voltammetry study of a gel polymer electrolyte based redox-capacitor. Sri Lankan Journal of Physics. 16, 19-27

Hashmi S 2004. Super capacitor: an emerging power source. National Academic Sci. Lett. 27, $27-46$ 
Hashmi SA, Kumar A. Tripathi SK 2005. Investigations on electrochemical supercapacitors using polypyrrole redox electrodes and PMMA based gel electrolytes. European Polymer Journal. 41, 1373-1379

Ingram MD, Staesche H, Ryder KS 2004. Ladder doped polypyrrole: a possible electrode material for inclusion in electrochemical super capacitors. Journal of Power Sources. 129, 107-112

Jayathilake YMCD, Perera KS, Vidanapathirana KP, Bandara LRAK 2014. A novel gel polymer electrolyte based on polymethylmethacrylate and copper trifluromethanesulfonate. Journal of Electroanalytical Chemistry. 724, 125-129

Jayathilake YMCD, Perera KS, Vidanapathirana KP 2015. Preparation and characterization of a polyacrylonitrile-based gel polymer electrolyte complexed with 1 methyl-3 propyl immidazolium iodide. Journal of Solid State Electrochemistry. 19 (8), 2199-2203

Kumar D, Hashmi SA 2010. Ionic liquid based sodium ion conducting gel polymer electrolytes. Solid State Ionics. 181, 416-423

Liu Z, Abedin SZE, Endres F 2014. Electrodeposition and stripping of Zinc from an ionic liquid polymer gel electrolyte for rechargeable zinc based batteries. Journal of Solid State Electrochemistry. 18, 2581-2587

Pandey GP, Rastogi AC, Westgate CR 2013. Polyacrylonitrile and 1ethyl 3 methyl imidazolium thiocyanate based gel polymer electrolyte for solid state supercapacitors with grapheme electrodes. ECS Transactions. 50(43) 145-151

Perera K, Vidanapathirana KP, Dissanayake MAKL 2007. Artificial muscles based on Polyacrylonitrile based electrolytes. Sri Lankan Journal of Physics. 8, 39-45

Perera K, Vidanapathirana KP, Dissanayake MAKL 2011. Effect of the polymer host, Polyacrylonitrile on the performance of Li rechargeable cells. Sri Lankan Journal of Physics. 12, 25-31

Prabhaharan SRS, Vimala R, Zainal Z 2006. Nano structured mesoporous carbon as electrodes for super capacitor. Journal of Power Sources. 161, 730-736

Ramesh S, Lu SC, Morris E 2012. Towards magnesium ion conducting poly(vinylidenefluoride-hexafluoropropylene) based solid polymer electrolytes with great prospects : ionic and dielectric behaviour. Journal of Taiwan Institute of Chemical Engineers 43, 806-812

Ravindran D, Vickraman P 2012. Magnesium ionic conductivity behaviour of mixed salt system in PVA-PEG blend matrix. International Journal of Science and Research Publications. 2112, 1-4

Sharma JP, Sekhon SS 2006. PMMA based polymer gel electrolytes containing $\mathrm{NH}_{4} \mathrm{PF}_{6}$ : Role of molecular weight of polymer. Materials Science and Engineering B. 129, 104-108

Sun A, Xu Y, Wang J 2012. Electropolymerized composite film of polypyrrole and functionalized multi walled carbon naotubes: effect of functionalization time on capacitve performance. Journal Solid State Electrochemistry. 16, 1781-1789

Uppugalla S, Male U, Srinivasan P 2014. Design and synthesis of heteroatoms doped carbon / polyaniline hybrid material for high performance electrode in super capacitor application. Electrochimica Acta. 146, 242-248

Wang W, Guo S, Penchev M, Ruiz I, Bozhilov KN, Yan D, Ozkan M, Ozkan CS 2013. 3d few layer grapheme and carbon nano tube foam architectures for high fidelity super capacitors. Nano Energy, 2, 294-303

Wang Y, Yang Y, Zhang X, Liu C, Hao X 2015. One step electrodeposition of polyaniline / nicle hexacynoferrate sulfonated carbon nano tubes interconnected composite films for super capacitors, Journal of Solid State Electrochemistry, 19, 3157-3168

Wu TY, Li WB, Kuo CW, Chou CF, Liao JW, Chen HR, Tseng CG 2013. Study of PMMA based gel electrolyte for EC devices. International Journal of Electrochem Science, 8, 10720-10732 